\title{
Antidepressant Fluoxetine Does Not Appear to Interfere With Key Translational Parameters in the Rat Adjuvant-induced Arthritis Model
}

\author{
DASA SEVELJEVIC-JARAN ${ }^{1,2}$, OTTO KALLIOKOSKI ${ }^{2}$, KLAS S.P. ABELSON ${ }^{2}$ and JANN HAU ${ }^{2}$ \\ ${ }^{1}$ Toxicology and Laboratory Animal Science Department, Fidelta Ltd., Zagreb, Croatia; \\ ${ }^{2}$ Department of Experimental Medicine, Faculty of Health and Medical Sciences, \\ University of Copenhagen, Copenhagen, Denmark
}

\begin{abstract}
Background/Aim: This study aimed to investigate the analgesic effects of fluoxetine on Lewis rats of both sexes in the adjuvant-induced arthritis (AIA) rat model. In humans, chronic pain syndromes typical of rheumatoid arthritis $(R A)$ co-exist with depression which is often treated with fluoxetine antidepressant known to have antinociceptive effects. Materials and Methods: The experiment was terminated on day 26, after seven days of oral treatment (days 19-25) with fluoxetine and indomethacin. The effects of treatments were assessed on the final day of the study through measuring body weight, serum concentrations of a1acid glycoprotein, visual arthritis assessment and post mortem histopathology assessment. Results: Statistically significant difference was determined in the body weight of male subjects, with indomethacin-treated animals putting on significantly more weight than the vehicle and fluoxetinetreated counterparts. No differences were found between the different treatment groups in other study assessments. Conclusion: The present study did not provide support for analgesic effects of fluoxetine aimed at reducing the severity of the AIA model.
\end{abstract}

Rheumatoid arthritis (RA) is a painful, chronic, autoimmune disease which affects around $2 \%$ of the worldwide population (1). Research and development of treatments of arthritis currently involve the use of animal models, such as the rat adjuvant-induced arthritis (AIA) model. This model is classified as a severe model, inflicting long-lasting pain

This article is freely accessible online.

Correspondence to: Dasa Seveljevic-Jaran, DVM, MSc LAS, Toxicology and Laboratory Animal Science Department, Fidelta Ltd., 10000 Zagreb, Croatia. E-mail: dasa.seveljevicjaran@fidelta.eu

Key Words: Rheumatoid arthritis, rat model, fluoxetine, analgesia. on the animals. Inflicting pain on experimental animals is problematic $(1,2)$. Firstly, it poses an ethical issue, since the studied animals' well-being is compromised. Secondly, it is scientifically problematic, since much of the pain may be unnecessary, not contributing to the modelling of the disease. Instead, the pain may provoke unintended physiological and endocrine changes in the animal, confounding results and compromising the validity of the experiment (2-4). Consequently, it is important to refine procedures and interventions that may cause unnecessary pain and stress in the studied animals. It is desirable to treat pain in a manner that will not negatively affect the translational properties of the model.

Inflammation and its associated pain co-exist as clinical symptoms in both human arthritis and the rat adjuvantinduced arthritis model and have been reported to co-occur with depression or with depression-like-behaviour, respectively (5-8). Depression and persistent pain symptoms exacerbate one another, which has made some researchers refer to the comorbidity as the pain-depression syndrome (8, 9). Antidepressants have been used in patients suffering from chronic pain primarily to treat concomitant depression, however, clinical and preclinical studies have repeatedly demonstrated analgesic effects of antidepressants, separate from their antidepressant activities $(5-7,10,11)$.

The antidepressant drug fluoxetine is a selective serotonin reuptake inhibitor (SSRI) which has been documented to have anti-inflammatory and analgesic effects in addition to its antidepressant effects in humans and rats $(5,8,12-14)$. With this in mind, the present study investigated whether fluoxetine treatment would influence the classical parameters usually assessed in the rat AIA model. The effects of fluoxetine were studied in male and female Lewis rats in the chronic phase of disease progression (19-25 days after induction). Inflammation and affectedness were assessed through measuring body weight and serum concentrations of $\alpha 1$-acid glycoprotein, through visual arthritis assessment and 
post mortem histopathology assessment. The nonsteroidal anti-inflammatory drug (NSAID) indomethacin, which is frequently used for symptomatic treatment of RA in humans and animals, was used as a positive control (15-19).

\section{Materials and Methods}

Ethical statement. All experiments were carried out in an Association for Assessment and Accreditation of Laboratory Animal Care International-accredited facility under the supervision of an Institutional Ethics Committee in collaboration with the Croatian Competent Authority and the National Ethics Committee, granting this project 2-year authorisation (authorisation number KLASA: UP/I-322-01/17-01/162, URBROJ: 525-10/0255-18-4) in compliance with the 2010/63/EU Directive requirements.

Animals and housing. Seven-week-old male $(\mathrm{n}=38)$ and female $(\mathrm{n}=38)$ SPF Lewis rats (LEW/Crl; Charles River, Sulzfeld, Germany) were pair-housed in open-top cages $(1291 \mathrm{H} ; 425 \times 266 \times 185 \mathrm{~mm}$; Tecniplast, Buguggiate, Italy) on arrival from the breeder. The animal holding rooms within a BSL2 Barrier Unit were maintained at $22 \pm 2^{\circ} \mathrm{C}$, and $55 \pm 10 \%$ relative humidity, with $\geq 15$ air changes per hour. A diurnal rhythm was kept through a 12:12 h dark/light cycle (lights on at 7:00 am), with a daytime intensity of 150 Lux at $1 \mathrm{~m}$ over the floor. The conventional cages (Tecniplast Type III H) were equipped with raised lids, bedding consisted of corn cob grit (Scobis Due; Mucedola, Milan, Italy), and enrichment items were provided in the form of cardboard shelters and wooden gnawing blocks (LBS Biotechnology, Horley, UK). Feed pellets (VRF1; SDS Diets, Horley, UK) and tap water were provided ad libitum. Pellets were also placed on the bedding for easier access for arthritic animals. Cages were changed twice a week, transferring only a handful of used bedding to maintain olfactory cues.

Experimental design. All procedures were carried out in a randomized order and the experimenters were blinded to the identity of the animals throughout.

Following 5-7 days of acclimatization, arthritis was induced through adjuvant injections - designated as day 1 of the experiment. On day 19 , only the arthritic animals were randomly assigned to receive daily treatments of $1 \mathrm{mg} / \mathrm{kg}$ bodyweight indomethacin, 20 $\mathrm{mg} / \mathrm{kg}$ bodyweight fluoxetine treatment, or vehicle. Healthy control animals, neither arthritic nor receiving any treatment, were included in the study. Each experimental group consisted of eight males and eight females. The experiment was terminated on day 26 , after 7 days of treatments.

Disease induction. Freund's complete adjuvant was prepared by suspending $10 \mathrm{mg} / \mathrm{ml}$ heat-inactivated Mycobacterium tuberculosis (Myc $t$, Difco H37 Ra; Fisher Scientific, Hampton, VA, USA) in an oil vehicle (Freund's adjuvant, incomplete, prod. no. F5506; SigmaAldrich, St. Louis, MO, USA). Rats were anesthetized with $4 \%$ isoflurane (Forane, Abbot, Chicago, IL, USA) delivered in pure oxygen in an induction chamber. Anaesthesia was maintained through a face mask while a small area of the lower back was shaved with electric clippers. A $100 \mu$ injection of the adjuvant was administered intradermally, approximately $1 \mathrm{~cm}$ cranially to the tail base, before the subject was returned to its cage for recovery. On day 18 post-induction, rats not developing early stages of arthritis were excluded from the study. These non-responders were identified based on body weight (gain), absence of bilateral midfoot oedema and lack of a visible limp when allowed to explore the flat surface of a bench.

Treatments. All formulations were prepared fresh every day. Indomethacin $(0.1 \mathrm{mg} / \mathrm{ml}$; Sigma-Aldrich) and fluoxetine $(2 \mathrm{mg} / \mathrm{ml}$; Sigma-Aldrich) were dissolved in an aqueous solution of $0.5 \%$ $(\mathrm{w} / \mathrm{v})$ methyl cellulose (Sigma-Aldrich). A solution of $0.5 \%$ methyl cellulose was used as a vehicle for (negative) control animals. Treated animals were dosed daily using a 5 -cm curved stainless steel gavage needle with a ball tip. All prepared solutions were administered orally at $10 \mathrm{ml} / \mathrm{kg}$ bodyweight.

Clinical assessments. In addition to routine welfare monitoring, the animals' disease progression was followed throughout the study. The animals were weighed on days $1,5,10,15,18,22$ and 25. On day 19, at the start of therapy, and on the final day of the experiment, the animals' hind paws were photographed (two standardized digital photographs showing bottom/plantar and lateral views of both hind paws, respectively) so as to ensure the blinded assessment of arthritis. Using these images, the number of clearly arthritic joints were scored by four independent assessors (total score of 24 for two hind paws) in accordance with Hawkins et al. visual assessment scoring system (20).

Biomarkers. Blood samples were collected on days 1, 5, 10, 18 and 26 . The sampled rat was moved to a warming cabinet $\left(39^{\circ} \mathrm{C}\right)$ for 10 min after which its tail vein was punctured and a blood sample of approximately $250 \mu \mathrm{l}$ was obtained. Serum was separated and stored at $-80^{\circ} \mathrm{C}$ until analysis. Samples were analyzed for tumor necrosis factor alpha (TNF $\alpha)$ and $\alpha_{1}$-acid glycoprotein $\left(\alpha_{1}\right.$ AGP) content using commercially available enzyme-linked immunosorbent assay kits (rat TNF $\alpha$ DuoSet; R\&D Systems, Minneapolis, MN, USA; Rat $\alpha 1$-AGP; MyBioSource, San Diego, CA, USA). However, TNF $\alpha$ concentrations were below the detection limit throughout, and were consequently omitted from the present report.

Histopathology. On day 26, the rats were euthanized by exsanguination under surgical anaesthesia delivered i.p. (100 mg/kg ketamine, $5 \%$ solution for injection; Narkamon, Bioveta, Ivanovice na Hané, Czech Republic) and $20 \mathrm{mg} / \mathrm{kg}$ xylazine (Xylazine 2\% solution for injection; Alfasan, JA Woerden, the Netherlands). Both hind paws, severed at the level of the ankle, were submerged in $10 \%$ neutral buffered formalin for 24 hours at room temperature (Thermo Shandon Ltd., subsidiary of Thermo Fisher Scientific, Cheshire, UK). After 24 hours, the paws were transferred to a decalcifying solution of $10 \%$ formic acid (Honeywell Fluka, Charlotte, NC, USA) in $10 \%$ neutral buffered formalin (Thermo Shandon Ltd.) where they were left submerged for 10 days. Subsequently, the joints were cut, mid-longitudinally, through the center, and embedded in paraffin. Microtome sections of 3-4 $\mu \mathrm{m}$ were prepared and stained using hematoxylin-eosin. The slides were assessed by two pathologists, independently. Combined scores grading inflammation, bone erosion and osteoclast activity were employed (Table I).

Statistical analysis. For hypothesis tests, only arthritic animals were included. Consequently, fluoxetine-treated animals were compared to vehicle-treated animals (negative controls) and indomethacintreated animals (positive controls). Continuous variables, namely body weight on the final day of the study and serum $\alpha_{1}$ AGP 
Table I. Histopathological scoring of arthritic severity. The scores were developed by Bolon et al. (21) and Coxon et al. (22).

\begin{tabular}{|c|c|c|}
\hline & & Score \\
\hline \multirow[t]{5}{*}{ Inflammation } & None. Normal tissue. & 0 \\
\hline & Few inflammatory cells. & 1 \\
\hline & Mild inflammation. & 2 \\
\hline & $\begin{array}{l}\text { Moderate inflammation (often, } \\
\text { but not always, diffuse) }\end{array}$ & 3 \\
\hline & $\begin{array}{l}\text { Marked inflammation (diffuse and dense, } \\
\text { with large periarticular abscesses). }\end{array}$ & 4 \\
\hline \multirow[t]{9}{*}{ Bone erosion } & None. Normal tissue. & 0 \\
\hline & $\begin{array}{l}\text { Minimal loss of cortical or trabecular } \\
\text { bone at a few sites. }\end{array}$ & 1 \\
\hline & $\begin{array}{l}\text { Mild loss of cortical or trabecular bone at } \\
\text { modest number of sites (generally tarsals). }\end{array}$ & 2 \\
\hline & Moderate loss of bone at many sites. & 3 \\
\hline & $\begin{array}{l}\text { Usually the trabeculae of the tarsals, } \\
\text { but sometimes the cortex of the distal tibia. }\end{array}$ & \\
\hline & Marked loss of bone at many sites. & 4 \\
\hline & $\begin{array}{l}\text { Usually as extensive destruction } \\
\text { of trabeculae in the tarsals, but sometimes } \\
\text { with partial loss of cortical bone } \\
\text { in the distal tibia. }\end{array}$ & \\
\hline & Marked loss of bone at many sites. & \\
\hline & $\begin{array}{l}\text { With fragmenting of tarsal trabeculae } \\
\text { and full-thickness penetration } \\
\text { of cortical bone in the distal tibia. }\end{array}$ & 5 \\
\hline \multirow[t]{5}{*}{ Osteoclasts } & No osteoclasts. Normal tissue. & 0 \\
\hline & $\begin{array}{l}\text { Few osteoclasts (lining less than } 5 \% \\
\text { of the most affected bone surfaces). }\end{array}$ & 1 \\
\hline & $\begin{array}{l}\text { Some osteoclasts (lining } 5-25 \% \\
\text { of the most affected bone surfaces). }\end{array}$ & 2 \\
\hline & $\begin{array}{l}\text { Many osteoclasts (lining } 25-50 \% \\
\text { of the most affected bone surfaces). }\end{array}$ & 3 \\
\hline & $\begin{array}{l}\text { Myriad osteoclasts (lining more than } 50 \% \\
\text { of the most affected bone surfaces). }\end{array}$ & 4 \\
\hline
\end{tabular}

concentrations, were compared using simple one-way analysis of variance. Visual scores were compared using the Kruskal-Wallis test. Hypothesis test outcomes of $p<0.05$ were considered statistically significant throughout.

\section{Results}

Two weeks after induction, the body weights of the nowarthritic animals deviated from those of healthy controls (Figure 1). Following daily treatments, a larger variation in body weights was seen in the arthritic animals on the final day of the study. The difference, however, was only statistically significant in the males $\left(\mathrm{F}_{2,21}=6.81, p=0.005\right)$, with indomethacin-treated animals gaining significantly more weight than the vehicle-treated animals (Tukey's HSD: $p=0.033)$ and fluoxetine-treated animals $(p=0.006)$. A similar trend was not seen for females $\left(\mathrm{F}_{2,21}=1.92, p=0.17\right)$.

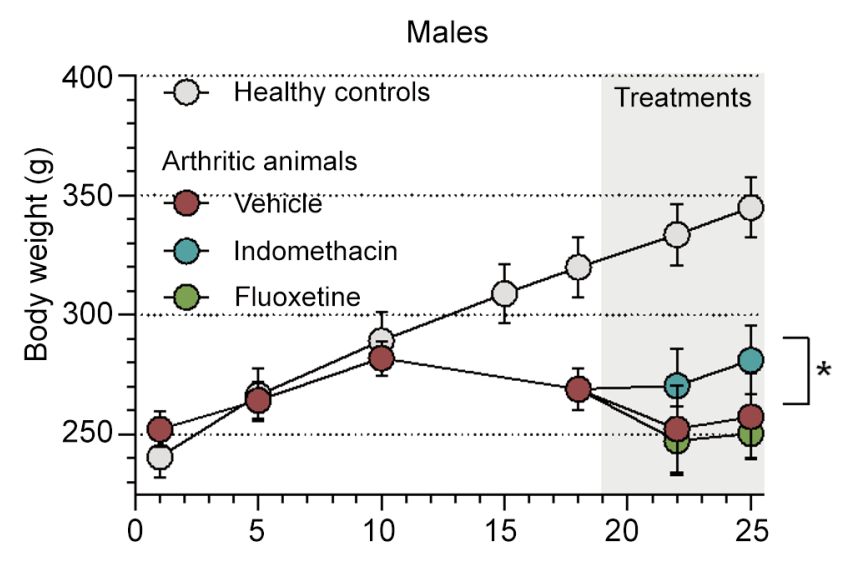

Females

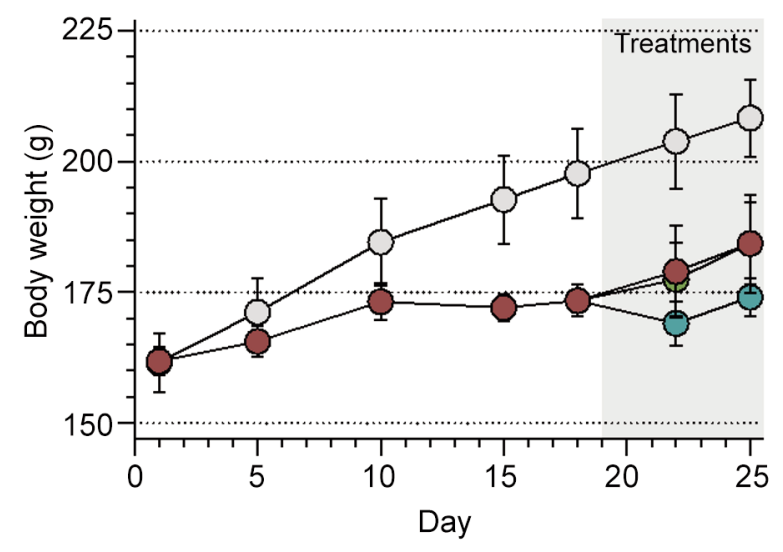

Figure 1. Body weight curves for male and female rats. All experimental groups consisted of eight animals. Data are presented as the mean $\pm 95 \%$ confidence interval. *Statistically significant difference at $p<0.05$.

Visual arthritis scores revealed no statistically significant difference in the average number of affected joints between treated males $\left(\mathrm{H}_{2}=1.1, p=0.58\right)$ or females $\left(\mathrm{H}_{2}=0.29\right.$, $p=0.86)$. The arthritic animals were clearly affected, however, when compared to the healthy controls (Figure 2).

Most of the stored aliquoted serum samples were lost for subsequent $\alpha_{1}$ AGP analysis, except for serum samples collected from all females (arthritic and healthy controls) on day 26 and from males collected on days 19 and 26 (except the healthy controls). Samples were either exhausted during TNF $\alpha$ analyses or became desiccated due to prolonged inadequate freezer storage conditions. From the samples analysed, no statistically significant differences in serum $\alpha_{1}$ AGP concentrations were found between the different treatment groups for either sex (males: $\mathrm{F}_{2,20}=0.19, p=0.83$; females: $\mathrm{F}_{2,20}=0.47, p=0.63$ ). There was, however, a clear difference between healthy controls and arthritic animals in females; the serum samples for healthy control males were unfortunately lost, as stated above. Moreover, the circulating $\alpha_{1}$ AGP concentration in females was approximately twice as high as in males (Figure 3 ). 

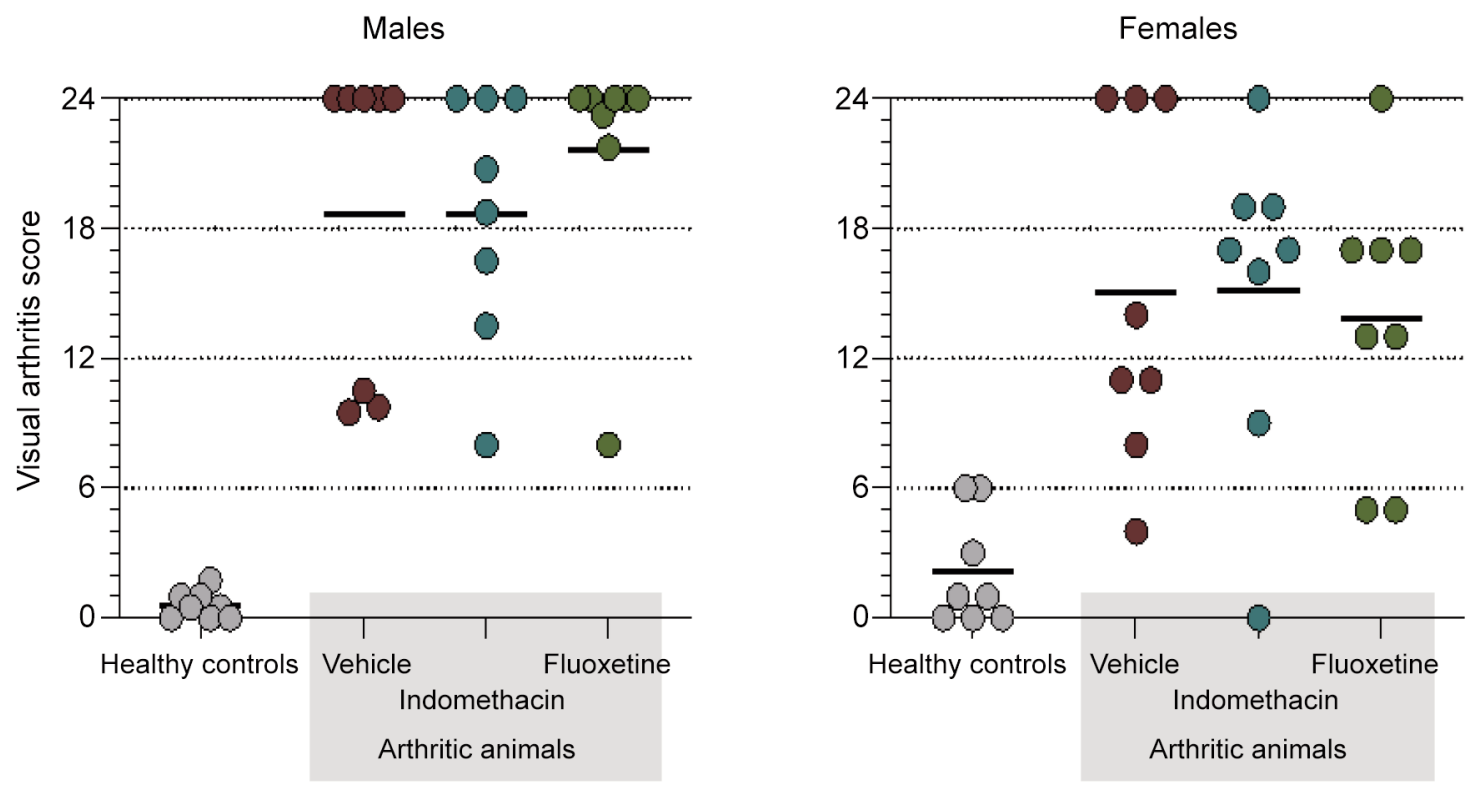

Figure 2. Visual arthritis scores for rats after 7 days of daily treatments with $1 \mathrm{mg} / \mathrm{kg}$ bodyweight indomethacin, $20 \mathrm{mg} / \mathrm{kg}$ bodyweight fluoxetine, or vehicle. Scores represent the number of arthritic/swollen joints, averaged between four observers. All experimental groups consisted of eight animals. Bars represent averages.

Males

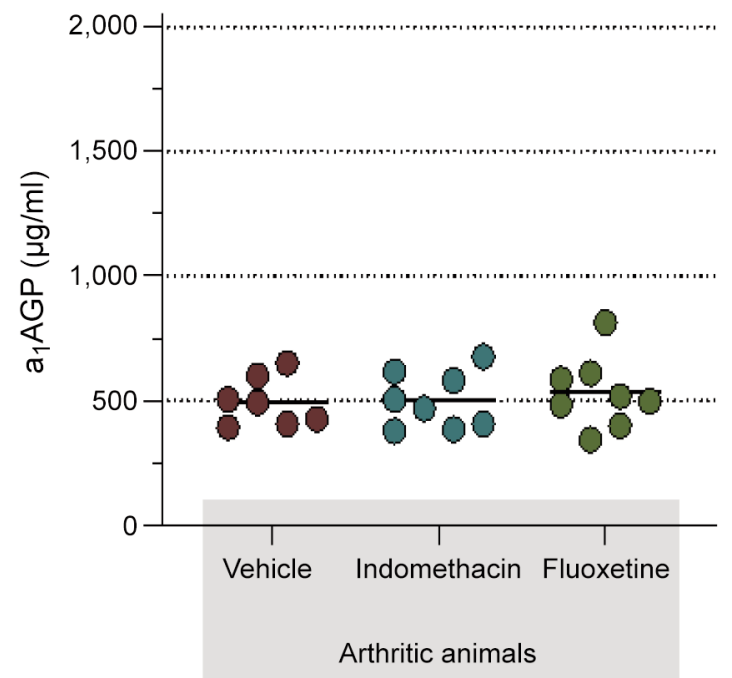

Females

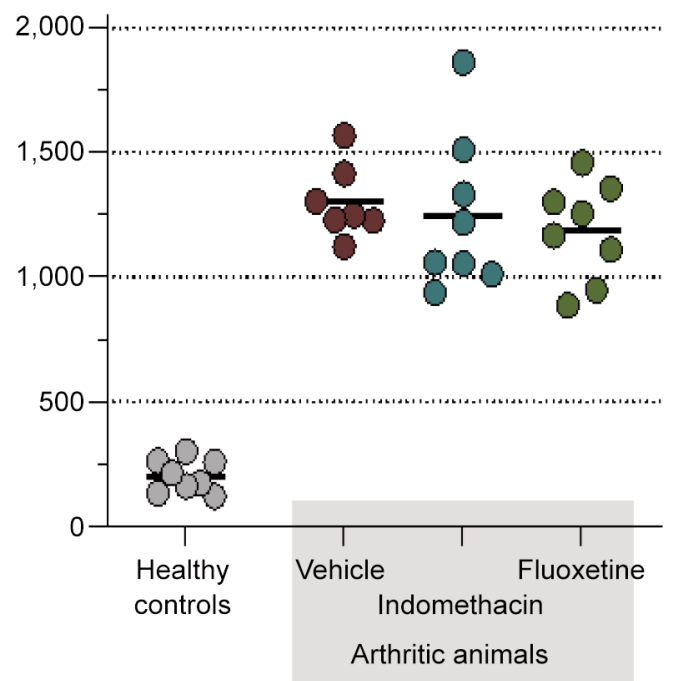

Figure 3. Serum $\alpha_{1}$-acid glycoprotein $\left(\alpha_{1} A G P\right)$ concentrations in rats after 7 days of daily treatments with $1 \mathrm{mg} / \mathrm{kg}$ bodyweight indomethacin, 20 $\mathrm{mg} / \mathrm{kg}$ bodyweight fluoxetine, or vehicle. All experimental groups consisted of eight animals. All samples for male healthy controls, one sample in the male vehicle-treated group and one in the female vehicle-treated group were lost prior to analysis. Bars represent averages.

Histological evaluation of the affected paws revealed no statistically significant differences between the treatment groups (males: $\mathrm{H}_{2}=0.44, p=0.80$; females: $\mathrm{H}_{2}=2.5, p=0.29$ ).
Much like the visual assessments, there was, however, a clear difference between the arthritic animals and healthy controls (Figure 4). 

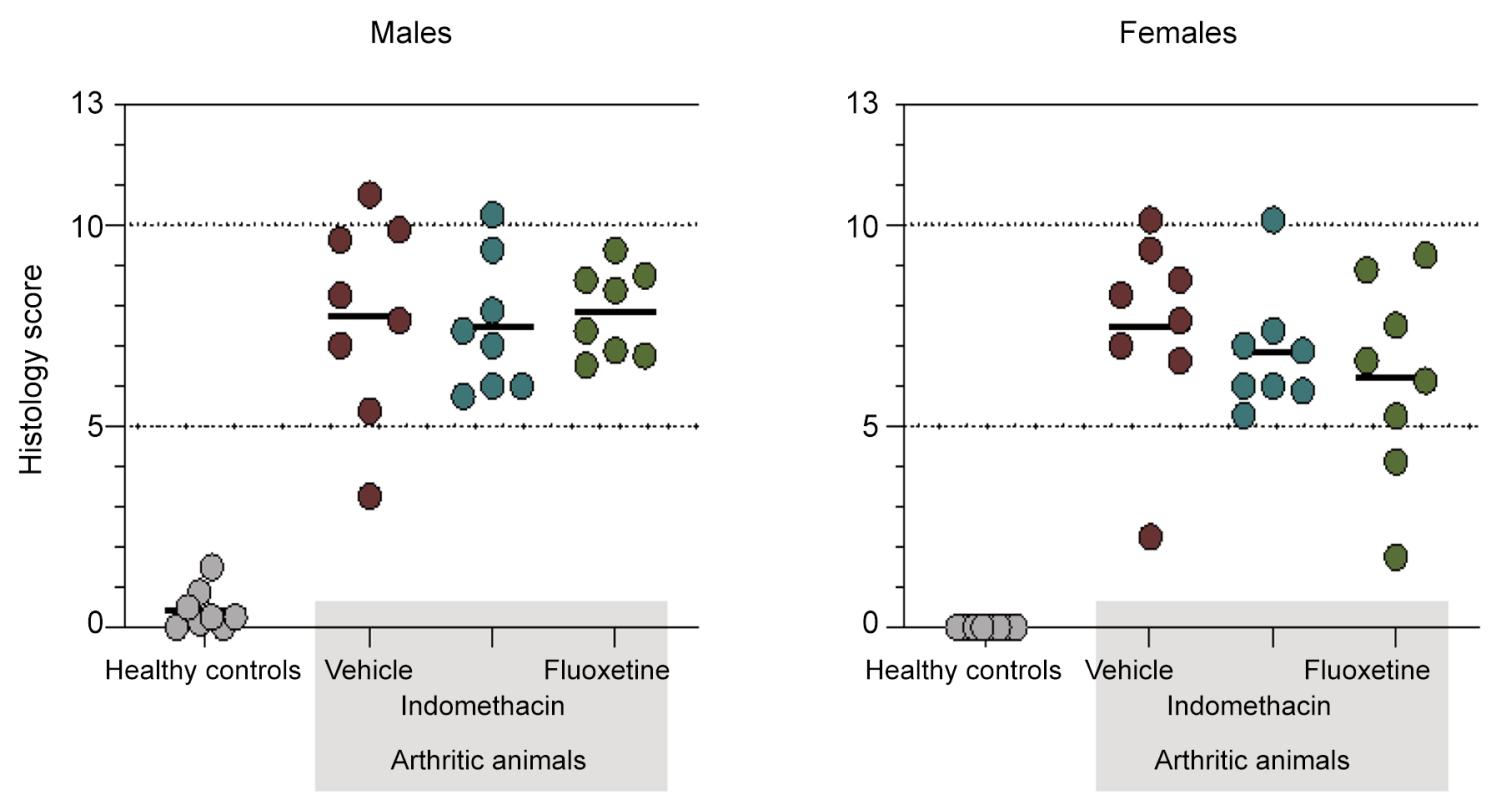

Figure 4. Histological scores assessing inflammation, bone erosion and osteoclasts in rats after 7 days of daily treatments with $1 \mathrm{mg} / \mathrm{kg}$ bodyweight indomethacin, $20 \mathrm{mg} / \mathrm{kg}$ bodyweight fluoxetine, or vehicle. Higher scores indicate a greater degree of inflammation and bone remodelling. All experimental groups consisted of eight animals. Bars represent averages.

\section{Discussion}

In humans suffering from RA, clinical symptoms of inflammation and its associated pain co-exist with depressive behaviour $(5,6,11)$. Historically, antidepressants have been used to treat pain in humans with the primary aim of treating concomitant depression. However, more recent clinical and preclinical studies have repeatedly demonstrated that the analgesic effects of antidepressants are separable from their antidepressant activities $(5,6,8,10-12)$.

In animal models of painful human conditions, pain management practices routinely used on humans are often neglected $(20,23)$. This neglect extends to the potentially confounding effects of untreated pain making it a poorly controlled pre-clinical study variable and a source of bias. The impact of analgesia on study results, in comparison, is more predictable than the effects of untreated pain in in vivo research $(2-4,24)$.

Pain is always the first symptom that patients with RA report when seeking medical help and it gets immediate relief treatment, long before the diagnosis of the disease is made, and optimal choice of therapeutics prescribed. In the clinic, it would be considered unethical and malpractice to withhold analgesics from human patients suffering from conditions that cause pain. Even in clinical trial contexts, analgesics are never withheld, and these are subjected to their potential confounding influences while evaluating the efficacy of novel drugs. Yet good practices from the clinic are rarely translated to the preclinical setting for fear that they may confound the results.

In the preclinical context, it should be considered that the translatability of animal models may be improved if analgesics are administered and used in a manner that more closely matches human treatment $(8,15,23,25)$.

RA is an autoimmune progressive disorder that preferentially affects peripheral joints. Patients with RA who present with signs and symptoms have disease manifestations comparable with active, chronic disease in rodent models $(15,26)$.

In humans, autoimmune diseases such as RA affect women more often than men, the sex ratio typically being around 3:1, most likely due to genetic (X-linked) factors and hormonal aspects (27). Although preclinical models of RA are helpful, there is an ongoing debate about the optimum model for particular disease aspects, as well as their translatability to human disease, with different models possessing different mechanistic and clinical features $(16,24$, 26, 28).

The rat AIA was the first animal model of RA to be described and it is still widely used in the preclinical testing of new drug candidates for arthritis. Although the mechanisms of arthritis induction are often not clear, the model is characterised by an initial immune-mediated inflammatory phase followed by joint destruction and ankylosis in the chronic phase of disease development $(26,28,29)$. 
Prototypic NSAID indomethacin was used here in the treatment of chronic inflammatory pain in this model of RA; NSAIDs are generally thought to be therapy of symptoms rather than disease-modifying, effective in treating the pain associated with arthritis but not inhibiting the progression of joint damage. NSAIDs have repeatedly demonstrated efficacy in rodent models of arthritis by, for example, reducing paw oedema, preventing weight loss, and affecting the concentration of disease-relevant biomarkers (15-17, 26, 29-34).

In the clinic, co-existing depression is often treated with fluoxetine which has also been proven to have antinociceptive effects in chronic pain syndromes typical of RA, which is why fluoxetine was an obvious novel candidate for the treatment of pain in this study $(5,6,14)$.

Apart from relying on analgesics and antiarthritic therapy, in this study we also implemented nonpharmacological refinements to pain management so as to enhance animal wellbeing, such as empathetic handling (removing the rats from the cage by supporting their body), easier access to food (pellets scattered on the cage floor), ensuring warmth through social housing and provision of shelter and deep bedding, and humane endpoints $(20,24,30,31,33-36)$.

The protective, anti-inflammatory effect of indomethacin was reflected in the significantly higher body weights of males only on the last day of the study when compared to vehicle or fluoxetine-treated males; on other translational parameters, indomethacin was without effect.

Visual scoring of paw oedema used in this study, although blindly performed, relied on formalized subjective semiquantitative scores that were defined, validated and published by researchers, instead of relying on standardized precision tools that enable more precise measurements of changes in paw volume (20). The units set by researchers are often open to subjective judgements by assessors, which undoubtedly increases the variability of scores between different assessors because subtle changes in the paw volumes of small laboratory rodents often escape even the trained human eye, which makes the assessment all the more challenging and certainly less precise (37-39). Although the risk of subjectivity in this study was minimised through blinding, for similar future research we recommend visual scoring be combined whenever possible, with more precise quantitative measurements, which can typically be obtained by water plethysmography or caliper measurements.

Fluoxetine treatment did not ameliorate the symptoms of AIA in rats and induced a slightly higher bodyweight loss in the arthritic males compared to other treatments. During the seven days of therapy, following the gavage, the bitter tasting fluoxetine formulation caused the rats to repeatedly engage in aversive behaviour such as increased oral grooming and burrowing-like activities $(40,41)$. Since we did not monitor daily food consumption across treatment groups, we can only make an educated assumption that the bitterness of fluoxetine possibly negatively affected the palatability of food and consequently rats' appetites during the last week of the study, thus contributing to the body weight loss in male rats. In future studies, this adverse effect might possibly be avoided by administering the fluoxetine formulation i.p., bearing in mind that repeated i.p. administrations hold the potential to increase the cumulative severity of this painful disease model, outweighing the potential benefits of parenteral fluoxetine administration.

On the last day of the therapy, no differences in serum $\alpha_{1}$ AGP concentrations were found between the different treatment groups for either sex. However, there was a difference in the $\alpha_{1}$ AGP level between females and males, the level in females being twofold that in males in the treatment groups. In most disease states, including inflammation, infection and cancer, $\alpha_{1}$ AGP behaves as an acute-phase protein which is detectable for several days after the stimulus, its levels reported to increase from 2- to 6-fold in humans and a much broader fold of induction in animals (from 2- to 20-fold depending on the animal species and the disease) (42).

In rats, $\alpha_{1} \mathrm{AGP}$ is the dominant acute-phase protein; in male Lewis rats modelling AIA, the serum $\alpha_{1}$ AGP levels in healthy and arthritic rats were reported to be 4- to 14-fold different, with $\alpha_{1}$ AGP peaking immediately before the onset of arthritis, after which it steadily declined towards the chronic phase of the disease $(42,43)$. There have been mixed reports regarding sex-related differences in $\alpha_{1}$ AGP levels, for example, elevated concentrations were recorded in adult women and female minipigs, but they were relatively modest compared to the differences observed in different disease states or different age groups $(44,45)$. Whether this finding is strain-specific needs to be confirmed in similar future experiments on the same rat strain with the same read outs.

To conclude, since the AIA rat model inflicts prolonged pain and suffering on animals, it is important to continuously search for possible refinement strategies for the model. The present study did not provide support for analgesic effects of fluoxetine aimed at reducing the severity of the AIA model but on the other hand, fluoxetine had no impact on disease progression and severity.

Animal pain, treated or not, can be a source of bias and appropriate pain management should be routinely implemented and transparently reported as a preclinical standard, thus ensuring greater translatability and reproducibility of animal experiments.

\section{Conflicts of Interest}

The Authors and Funders declare that they have no competing interests. 


\section{Authors' Contributions}

Dasa Seveljevic-Jaran confirms contribution to the study conception, design, data collection and analysis. All Authors confirm contribution to the interpretation of results and draft article preparation. Finally, all Authors reviewed the results and approved the final version of the article.

\section{Acknowledgements}

The Authors would like to thank Fidelta's managers, the peers and the technicians for providing much appreciated financial and technical assistance for all in vivo and ex vivo research activities.

\section{References}

1 Fischer BD, Adeyemo A, O'Leary ME and Bottaro A: Animal models of rheumatoid pain: experimental systems and insights. Arthritis Res Ther 19(1): 146, 2017. PMID: 28666464. DOI: 10.1186/s13075-017-1361-6

2 Carbone L: Pain in laboratory animals: the ethical and regulatory imperatives. PLoS One 6(9): e21578, 2011. PMID: 21915253. DOI: 10.1371/journal.pone.0021578

3 Carbone L and Austin J: Pain and laboratory animals: publication practices for better data reproducibility and better animal welfare. PLoS One 11(5): e0155001, 2016. PMID: 27171143. DOI: 10.1371/journal.pone.0155001

4 Jirkof P: Side effects of pain and analgesia in animal experimentation. Lab Anim (NY) 46(4): 123-128, 2017. PMID: 28328895. DOI: 10.1038/laban.1216

5 Perrot S, Javier RM, Marty M, Le Jeunne C, Laroche F and CEDR (Cercle d'Etude de la Douleur en Rhumatologie France), French Rheumatological Society, Pain Study Section: Is there any evidence to support the use of anti-depressants in painful rheumatological conditions? Systematic review of pharmacological and clinical studies. Rheumatology (Oxford) 47(8): 1117-1123, 2008. PMID: 18445628. DOI: 10.1093/rheumatology/ken110

6 Perrot S, Maheu E, Javier RM, Eschalier A, Coutaux A, LeBars $\mathrm{M}$, Bertin P, Bannwarth $\mathrm{B}$ and Trèves R: Guidelines for the use of antidepressants in painful rheumatic conditions. Eur J Pain 10(3): 185-192, 2006. PMID: 16490727. DOI: 10.1016/j.ejpain. 2005.03.004

7 Liu MG and Chen J: Preclinical research on pain comorbidity with affective disorders and cognitive deficits: Challenges and perspectives. Prog Neurobiol 116: 13-32, 2014. PMID: 24444673. DOI: 10.1016/j.pneurobio.2014.01.003

8 Li JX: Pain and depression comorbidity: a preclinical perspective. Behav Brain Res 276: 92-98, 2015. PMID: 24797835. DOI: 10.1016/j.bbr.2014.04.042

9 Bair MJ, Robinson RL, Katon W and Kroenke K: Depression and pain comorbidity: a literature review. Arch Intern Med 163(20): 2433-2445, 2003. PMID: 14609780. DOI: 10.1001/ archinte.163.20.2433

10 Baharav E, Bar M, Taler M, Gil-Ad I, Karp L, Weinberger A and Weizman A: Immunomodulatory effect of sertraline in a rat model of rheumatoid arthritis. Neuroimmunomodulation 19(5): 309-318, 2012. PMID: 22797111. DOI: 10.1159/000339109

11 Verdu B, Decosterd I, Buclin T, Stiefel F and Berney A: Antidepressants for the treatment of chronic pain. Drugs 68(18):
2611-2632, 2008. PMID: 19093703. DOI: 10.2165/0003495200868180-00007

12 Sacre S, Medghalchi M, Gregory B, Brennan F and Williams R: Fluoxetine and citalopram exhibit potent antiinflammatory activity in human and murine models of rheumatoid arthritis and inhibit toll-like receptors. Arthritis Rheum 62(3): 683-693, 2010. PMID: 20131240. DOI: 10.1002/art.27304

13 Abdel-Salam OM, Baiuomy AR and Arbid MS: Studies on the anti-inflammatory effect of fluoxetine in the rat. Pharmacol Res 49(2): 119-131, 2004. PMID: 14643692. DOI: 10.1016/j.phrs. 2003.07.016

14 Fishbain D: Evidence-based data on pain relief with antidepressants. Ann Med 32(5): 305-316, 2000. PMID: 10949061. DOI: $10.3109 / 07853890008995932$

15 Bevaart L, Vervoordeldonk MJ and Tak PP: Evaluation of therapeutic targets in animal models of arthritis: how does it relate to rheumatoid arthritis? Arthritis Rheum 62(8): 2192-2205, 2010. PMID: 20506322. DOI: 10.1002/art.27503

16 Bendele A, McComb J, Gould T, McAbee T, Sennello G, Chlipala E and Guy M: Animal models of arthritis: relevance to human disease. Toxicol Pathol 27(1): 134-142, 1999. PMID: 10367688. DOI: $10.1177 / 019262339902700125$

17 Sardar S and Andersson A: Old and new therapeutics for Rheumatoid Arthritis: in vivo models and drug development. Immunopharmacol Immunotoxicol 38(1): 2-13, 2016. PMID: 26769136. DOI: $10.3109 / 08923973.2015 .1125917$

18 DeMarco GJ and Nunamaker EA: A review of the effects of pain and analgesia on immune system function and inflammation: relevance for preclinical studies. Comp Med 69(6): 520-534, 2019. PMID: 31896389. DOI: 10.30802/AALAS-CM-19-000041

19 Suokas AK, Sagar DR, Mapp PI, Chapman V and Walsh DA: Design, study quality and evidence of analgesic efficacy in studies of drugs in models of OA pain: a systematic review and a meta-analysis. Osteoarthritis Cartilage 22(9): 1207-1223, 2014. PMID: 25008207. DOI: 10.1016/j.joca.2014.06.015

20 Hawkins P, Armstrong R, Boden T, Garside P, Knight K, Lilley E, Seed M, Wilkinson M and Williams RO: Applying refinement to the use of mice and rats in rheumatoid arthritis research. Inflammopharmacology 23(4): 131-150, 2015. PMID: 26168847. DOI: $10.1007 / \mathrm{s} 10787-015-0241-4$

21 Bolon B, Campagnuolo $G$ and Feige U: Duration of bone protection by a single osteoprotegerin injection in rats with adjuvant-induced arthritis. Cell Mol Life Sci 59(9): 1569-1576, 2002. PMID: 12440777. DOI: 10.1007/s00018-002-8530-7

22 Coxon A, Bolon B, Estrada J, Kaufman S, Scully S, Rattan A, Duryea D, Hu YL, Rex K, Pacheco E, Van G, Zack D and Feige $\mathrm{U}$ : Inhibition of interleukin-1 but not tumor necrosis factor suppresses neovascularization in rat models of corneal angiogenesis and adjuvant arthritis. Arthritis Rheum 46(10): 2604-2612, 2002. PMID: 12384918. DOI: 10.1002/art.10546

23 Clutton RE: An anglocentric history of anaesthetics and analgesics in the refinement of animal experiments. Animals (Basel) 10(10): 1933, 2020. PMID: 33096686. DOI: 10.3390/ani10101933

24 Peterson NC, Nunamaker EA and Turner PV: To treat or not to treat: The effects of pain on experimental parameters. Comp Med 67(6): 469-482, 2017. PMID: 29212578.

25 Cohen S and Beths T: Grimace scores: tools to support the identification of pain in mammals used in research. Animals (Basel) 10(10): 1726, 2020. PMID: 32977561. DOI: 10.3390/ ani10101726 
26 Hegen M, Keith JC Jr, Collins M and Nickerson-Nutter CL: Utility of animal models for identification of potential therapeutics for rheumatoid arthritis. Ann Rheum Dis 67(11): 1505-1515, 2008. PMID: 18055474. DOI: 10.1136/ard.2007.076430

27 van Vollenhoven RF: Sex differences in rheumatoid arthritis: more than meets the eye. BMC Med 7: 12, 2009. PMID: 19331649. DOI: $10.1186 / 1741-7015-7-12$

28 Vincent TL, Williams RO, Maciewicz R, Silman A, Garside P and Arthritis Research UK animal models working group: Mapping pathogenesis of arthritis through small animal models. Rheumatology (Oxford) 51(11): 1931-1941, 2012. PMID: 22427408. DOI: 10.1093/rheumatology/kes035

29 Bolon B, Stolina M, King C, Middleton S, Gasser J, Zack D and Feige U: Rodent preclinical models for developing novel antiarthritic molecules: comparative biology and preferred methods for evaluating efficacy. J Biomed Biotechnol 2011: 569068, 2011. PMID: 21253435. DOI: $10.1155 / 2011 / 569068$

30 Mogil JS: Social modulation of and by pain in humans and rodents. Pain 156 Suppl 1: S35-S41, 2015. PMID: 25789435. DOI: $10.1097 / 01 . j$.pain.0000460341.62094.77

31 Mogil JS: Laboratory environmental factors and pain behavior: the relevance of unknown unknowns to reproducibility and translation. Lab Anim (NY) 46(4): 136-141, 2017. PMID: 28328894. DOI: 10.1038/laban.1223

32 Mogil JS, Davis KD and Derbyshire SW: The necessity of animal models in pain research. Pain 151(1): 12-17, 2010. PMID: 20696526. DOI: 10.1016/j.pain.2010.07.015

33 Turner PV: Moving beyond the absence of pain and distress: focusing on positive animal welfare. ILAR J 60(3): 366-372, 2021. PMID: 33119093. DOI: 10.1093/ilar/ilaa017

34 Turner PV and Barbee RW: Responsible science and research animal use. ILAR J 60(1): 1-4, 2019. PMID: 31930313. DOI: 10.1093/ilar/ilz020

35 Smith AJ, Clutton RE, Lilley E, Hansen KEA and Brattelid T: PREPARE: guidelines for planning animal research and testing. Lab Anim 52(2): 135-141, 2018. PMID: 28771074. DOI: $10.1177 / 0023677217724823$

36 Percie du Sert N, Hurst V, Ahluwalia A, Alam S, Avey MT, Baker M, Browne WJ, Clark A, Cuthill IC, Dirnagl U, Emerson M, Garner P, Holgate ST, Howells DW, Karp NA, Lazic SE, Lidster K, MacCallum CJ, Macleod M, Pearl EJ, Petersen OH, Rawle F, Reynolds P, Rooney K, Sena ES, Silberberg SD, Steckler T and Würbel H: The ARRIVE guidelines 2.0: Updated guidelines for reporting animal research. BMC Vet Res 16(1): 242, 2020. PMID: 32660541. DOI: 10.1186/s12917-020-02451-y

37 Hróbjartsson A, Thomsen AS, Emanuelsson F, Tendal B, Hilden $\mathrm{J}$, Boutron I, Ravaud $\mathrm{P}$ and Brorson S: Observer bias in randomized clinical trials with measurement scale outcomes: a systematic review of trials with both blinded and nonblinded assessors. CMAJ 185(4): E201-E211, 2013. PMID: 23359047. DOI: $10.1503 /$ cmaj.120744
38 Meagher R: Observer ratings: Validity and value as a tool for animal welfare research. Applied Animal Behaviour Science 119(1-2): 1-14, 2020. DOI: 10.1016/j.applanim.2009.02.026

39 Clarke $\mathrm{T}$, Pluske $\mathrm{J}$ and Fleming $\mathrm{P}$ : Are observer ratings influenced by prescription? A comparison of Free Choice Profiling and Fixed List methods of Qualitative Behavioural Assessment. Applied Animal Behaviour Science 177: 77-83, 2019. DOI: $10.1016 /$ j.applanim.2016.01.022

40 Bhat MG, Jordt RM, Khan MA, Foley CE and Gilbertson TA: Validation of a rat behavioral avoidance model from a drug delivery perspective. Int J Pharm 303(1-2): 31-36, 2005. PMID: 16125347. DOI: 10.1016/j.ijpharm.2005.06.015

41 Damsch S, Eichenbaum G, Tonelli A, Lammens L, Van den Bulck K, Feyen B, Vandenberghe J, Megens A, Knight E and Kelley M: Gavage-related reflux in rats: identification, pathogenesis, and toxicological implications (review). Toxicol Pathol 39(2): 348-360, 2011. PMID: 21422261. DOI: 10.1177/ 0192623310388431

42 Petersen HH, Nielsen JP and Heegaard PM: Application of acute phase protein measurements in veterinary clinical chemistry. Vet Res 35(2): 163-187, 2004. PMID: 15099494. DOI: 10.1051/ vetres:2004002

43 Smith SA and Waters NJ: Pharmacokinetic and pharmacodynamic considerations for drugs binding to alpha-1-acid glycoprotein. Pharm Res 36(2): 30, 2018. PMID: 30593605. DOI: 10.1007/s 11095-018-2551-x

44 Stolina M, Bolon B, Middleton S, Dwyer D, Brown H, Duryea D, Zhu L, Rohner A, Pretorius J, Kostenuik P, Feige U and Zack D: The evolving systemic and local biomarker milieu at different stages of disease progression in rat adjuvant-induced arthritis. J Clin Immunol 29(2): 158-174, 2009. PMID: 18726678. DOI: 10.1007/s10875-008-9238-8

45 Stolina M, Schett G, Dwyer D, Vonderfecht S, Middleton S, Duryea D, Pacheco E, Van G, Bolon B, Feige U, Zack D and Kostenuik P: RANKL inhibition by osteoprotegerin prevents bone loss without affecting local or systemic inflammation parameters in two rat arthritis models: comparison with antiTNFalpha or anti-IL-1 therapies. Arthritis Res Ther 11(6): R187, 2009. PMID: 20003323. DOI: 10.1186/ar2879

Received December 3, 2021 Revised December 22, 2021 Accepted January 13, 2022 